\title{
Lipid Rafts and Keratinocyte Apoptosis: Regulation via Death Receptors and Akt
}

\author{
Robert Gniadecki $^{*}, 1$ and Yves Poumay ${ }^{2}$
}

\author{
${ }^{I}$ Department of Dermatology, University of Copenhagen, Bispebjerg Hospital, Bispebjerg Bakke 23, 2400 Copenhagen \\ $N V$, Denmark \\ ${ }^{2}$ Cell and Tissue Laboratory, URPHYM, Faculty of Medicine, University of Namur, 5000 Namur, Belgium
}

\begin{abstract}
Lipid rafts are cholesterol-rich plasma membrane domains that regulate signal transduction. Lipid rafts can be disrupted by perturbing cholesterol metabolism, either by inhibition of cholesterol synthesis (e.g. by statins), by cholesterol sequestration or removal from the membrane (e.g. by methyl- $\beta$-cyclodextrin or filipin III) or by substitution with cholesterol derivatives, which do not support raft assembly (e.g. 5-cholestene 5- $\beta$-ol). Work performed in our laboratories as well as by others documented that lipid raft disruption precipitates classic apoptosis in normal and transformed keratinocytes and inhibited cell proliferation. Two mechanisms seem to play a particularly important role. One is mediated via the membrane death receptors such as Fas and TRAIL. Cholesterol depletion causes a ligandindependent activation of these receptors and activation of caspases. The other is mediated via the survival kinase Akt.

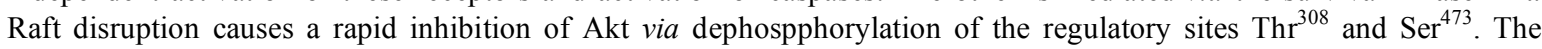
mechanism of this was the abrogation the binding of Akt and the major Akt kinase, PDK-1 to the membrane via pleckstrin homology (PH) domains. Diminished Akt activity results in deactivation of mTOR, activation of FoxO3a and an increased sensitivity to apoptotic stimuli. Thus, the integrity of lipid rafts is required for the activity of Akt and cell survival and may serve as a potential pharmacological target in the treatment of epidermal cancers.
\end{abstract}

The plasma membrane contains nanometer-large dynamic microdomains enriched in cholesterol, sphingolipids and gangliosides called "lipid rafts". These structures are important regulators of signal transduction, membrane geometry, and lateral movement of the molecules (see refs. [1-5] and elsewhere in this volume).

Since lipid rafts form spontaneously only in the narrow range of cholesterol concentration, the perturbation of membrane cholesterol disrupts lipid raft integrity and has been used extensively to study their role in signaling pathways. Of note, many receptors and enzymes, crucially involved in the regulation of cell survival and apoptosis have been reported to associate with rafts. For example, the epidermal growth factor receptor (EGFR), which proper function is a prerequisite for keratinocyte survival, can be transiently activated by cholesterol depletion due to increased ligand-independent dimerization and crossphosphorylation $[2,6]$ after release from the rafts. On the other hand, cholesterol depletion induces the activity of the stress kinase p38 [7] which signals towards cell cycle stop and increased differentiation of keratinocytes.

Old biochemical observations documented that cancer tissues contain more cholesterol than healthy tissue and it has been speculated that this may have functional significance for tumor growth [8]. Increased cholesterol content in the tissue and patients' sera have been found in

*Address correspondence to this author at the Department of Dermatology, University of Copenhagen, Bispebjerg Hospital, Bispebjerg Bakke 23, 2400 Copenhagen NV, Denmark; Tel: (+45) 35313165; Fax: (+45) 35315050; E-mail:rg01@bbh.regionh.dk actinic keratoses [9, 10]. Interestingly, cholesterol depletion from the membranes of leukemic cells and various other cancer cells resulted in apoptosis and increased sensitivity to chemotherapeutic drugs [11]. In this paper we review evidence that the same may hold true for normal and transformed human keratinocytes.

\section{DEPLETION OF MEMBRANE CHOLESTEROL CAUSES KERATINOCYTE APOPTOSIS}

Our laboratories have been the first to report that depletion of cholesterol in cultured keratinocytes causes apoptosis [12, 13]. These observations have been independently confirmed [14]. Elevated levels of cholesterol-rich lipid rafts in cancer cells are correlated with sensitivity to apoptosis induced by cholesterol-depleting agents [14]. Apoptotic keratinocytes in cholesterol-depleted cultures show the typical features of programmed cell death, including caspase activation, DNA fragmentation, nuclear shrinkage and formation of apoptotic bodies. Apoptosis has been induced by a variety of cholesterol-modifying agents such as methyl- $\beta$-cyclodextrin, statins, ceramides (which displace cholesterol from the rafts), filipin III (an agent that inactivates cholesterol in the membrane by complex formation) or cholesterol oxidase. However, a more detailed electron microscopic analysis of cholesterol-depleted cells revealed other interesting features, including a striking swelling of mitochondria, loss of mitochondrial potential $\Delta \Psi_{\mathrm{m}}$ and autophagocytosis ${ }^{1}$ It is thus possible that

\footnotetext{
${ }^{1}$ Gniadecki R, Wojewodzka U, Eefsen R, Gajkowska B (2005) Disruption of lipid rafts results in the caspase-independent, autophagic cell death. Poster no. 87, European Life Scientist Organization (ELSO) Meeting, Dresden, Germany; http:// www.elso.org/index.php?id=abstrlist2005\&lid=334).
} 
autophagocytic cell death is also involved in the cell death due to lipid raft disruption. This issue is currently investigated in our laboratory.

\section{CHOLESTEROL DEPLETION CAUSES A LIGAND- INDEPENDENT ACTIVATION OF DEATH RECEPTORS IN KERATINOCYTES}

Activation of membrane death receptors such as Fas or TRAIL constitutes one of the two major pathways leading to apoptosis. Under most circumstances, these receptors are activated upon binding of the cognate ligands, such as FasL (Fas ligand). There is extensive evidence that association with or dissociation from lipid rafts modulates the activatory state of death receptors [15]. In most cells, the activation of Fas takes place within the milieu of lipid rafts and raft disruption blocks Fas-dependent apoptosis [16, 17]

A theory has been forwarded suggesting that Fas, upon recruitment to rafts, forms large complexes with other components of the apoptotic machinery, including caspase- 8 and is further activated intracellularly precipitating apoptosis [18]. However, in some cell types, including epidermal keratinocytes and squamous cell carcinoma the opposite is true: depletion of cholesterol causes Fas clustering outside the rafts and activation of cell death pathway $[13,19]$. The mechanism seems to be similar: upon cholesterol depletion large membranous aggregates of Fas are observed which colocalize with caspase 8 and FADD (Fas-associated death domain protein). It seems that this activation is not dependent of the presence of ligand. Moreover, keratinocytes, which are slightly cholesterol depleted, are more sensitive to the death inducing ligands, such as FasL and TRAIL. Since carcinoma cells contain more cholesterol that normal cells, it is in principle possible to utilize this phenomenon for tumor sensitization to death ligands thus minimizing their systemic toxicities.

\section{AKT/PROTEIN KINASE B IS RESPONSIVE TO LIPID RAFT DISRUPTION AND MEDIATES KERATINOCYTE APOPTOSIS}

It is important to note that activation of surface death receptors is neither necessary nor sufficient for keratinocyte apoptosis after cholesterol depletion. Fas-induced apoptosis can normally be blocked by caspase- 8 inhibitors, but cholesterol-depleted keratinocytes undergo apoptosis even in the presence of this inhibitor (unpublished observations).

Akt/protein kinase $\mathrm{B}$ is a cardinal regulator of cell survival and its pathologic increase in activity causes carcinogenesis [20,21]. Membrane receptors including the ErbB family of surface receptors, IGFRs or Ras stimulate Akt kinase activity via the phosphoinositide 3-kinase (PI3K) $[22,23]$. PI3K catalyzes the conversion of membrane phosphatidylinositol 4-5-bisphosphate PIP2 to phosphatidylinositol 3,4,5-trisphosphate PIP3, to which both Akt and its immediate activator phosphatidylinositoldependent kinase 1 (PDK-1) bind via pleckstrin-homology $(\mathrm{PH})$ domains [24, 25]. Full activation of Akt requires phosphorylation of $\mathrm{Thr}^{308}$ by PDK-1, followed by a second phosphorylation at $\operatorname{Ser}^{473}$ [26]. Downstream components of the Akt-PI3K signaling cascade include mammalian target of rapamycin $(\mathrm{mTOR}) / \mathrm{p} 70^{\mathrm{S} 6 \mathrm{~K}}$ pathway $[27,28]$ and the apoptosis-related Forkhead familiy of transcription factors FoxO, causing their emigration from the nucleus [29, 30].

Lipid rafts are known to be enriched in PIP2 and PIP3 [31] and are therefore likely to provide the particularly permissive milieu for the interaction between PDK-1 and Akt. Indeed, Akt resides in the detergent-resistant (raft-like) membrane fraction. Using the fluorescence correlation spectroscopy technique Lasserre et al. [32] demonstrated that recruitment of Akt to lipid rafts facilitates its enzymatic activation. Guo et al. [33] confirmed these results using yet another technique of fluorescence resonance energy transfer with an artificially expressed Akt activity reporter. They showed a higher Akt activity with faster activation kinetics within lipid rafts and impaired Akt signaling in cholesteroldepleted cells. However, the activation of Akt in rafts seems to depend on both the cell type and the stimulus involved. In NIH 3 T3 cells used by Guo et al. [33] platelet-derived growth factor (PDGF) stimulated Akt in both raft and nonraft regions, albeit more efficiently in the raft domains. In contrast, insulin-like growth factor 1 (IGF-1) stimulates Akt that is localized in raft regions and in this case raft disruption by e.g. cholesterol depletion abolishes the total cellular Akt activity to a higher degree.

In keratinocytes and keratinocyte-derived cancer cell lines the activity of Akt is also dependent on the integrity of lipid rafts. Li et al. [14] showed that in A431 cells cholesterol depletion by methyl- $\beta$-cyclodextrin or simvastatin caused apoptosis accompanied by Akt deactivation. Confirming our previous observations [2] they showed that At deactivation occurs in spite of the fact that EGFR is activated. Our recent results ${ }^{2}$ confirm these findings. In both normal keratinocytes and keratinocte cell lines (HaCaT, A431) methyl- $\beta$-cyclodextrin and other cholesterol-active agents such as filipin III, cholesterol oxidase, simvastatin and 5 -cholesten-5- $\beta$-ol induced an abrupt dephosphorylation of Akt at its regulatory sites $\left(\mathrm{Thr}^{308}\right.$ and $\mathrm{Ser}^{473}$ ), inactivation of mTOR and nuclear export of FoxO3a. The mechanism of Akt deactivation in keratinocytes is reduction of the number of the phosphoinositide-enriched sites in the membrane to which Akt and PDK-1 bind via PH domains. Thus, in keratinocytes lipid rafts can function as reaction nanochambers allowing close proximity and enzymatic interaction between PDK-1 and Akt. Cholesterol perturbation causes disruption or dispersion of lipid rafts and movement of the growth factorPI3K complexes into the more fluid, non-raft areas of the membrane. As a consequence, the PIP3 produced by this enzyme is dispersed in the membrane instead of being concentrated in the highly organized raft nanodomains. As a consequence, the binding of $\mathrm{PH}$ domain proteins, such as Akt, PLC $\delta$ and PDK-1 to the membrane can be impaired which precludes their spatial interaction and Akt phosphorylation.

The discovery of the cholesterol-dependence of Akt in keratinocytes is likely to have practical consequences. Akt in keratinocytes constitutes a dominant survival pathway and its blockade leads to apoptotic and autophagic cell death,

\footnotetext{
${ }^{2}$ Damien Calay, Dina Vind-Kezunovic, Aurelie Frankart, Sylviane Lambert, Yves Poumay, Robert Gniadecki. Inhibition of Akt signaling by exclusion from lipid rafts in normal and transformed epidermal keratinocytes. Submitted
} 
even in the presence of an active EGFR-ERK axis. Decreased Akt activity renders the cells more susceptible to chemotherapeutics. The PI3K-Akt-mTOR pathway is hyperactivated in many types of cancer providing survival advantage to transformed cells [34] often due to inactivatory mutations in the gene coding PTEN lipid phosphatase (phosphatase and tensin homologue deleted on chromosome ten) mediating dephosphorylation of PIP3 to PIP2. Since cancer cells are enriched in caveolae and lipid rafts comparing to normal cells [14], pharmacologic cholesterol depletion may be a promising method for increasing chemosensitivity to the cytostatics in patients with malignant tumors. Preliminary observations on high dose statins support this notion [11].

\section{ACKNOWLEDGEMENTS}

Unpublished experimental data cited in this review were mostly generated by our colleagues: Damien Calay, Sylviane Lambert and Dina Vind-Kezunovic. This work was supported by grants from Aage Bangs Foundation and Jeppe Juhl and wife Ovita Juhls Foundation, Denmark.

\section{REFERENCES}

[1] Simons K, Toomre D. Lipid rafts and signal transduction. Nat Rev Mol Cell Biol 2000; 1: 31-9.

[2] Lambert S, Vind-Kezunovic D, Karvinen S, Gniadecki R. Ligandindependent activation of the EGFR by lipid raft disruption. J Invest Dermatol 2006; 126: 954-62.

[3] Vind-Kezunovic D, Nielsen CH, Wojewodzka U, Gniadecki R. Line tension at lipid phase boundaries regulates formation of membrane vesicles in living cells. Biochim Biophys Acta 2008; 1778: 2480-6.

[4] Vind-Kezunovic D, Wojewodzka U, Gniadecki R. Focal junctions retard lateral movement and disrupt fluid phase connectivity in the plasma membrane. Biochem Biophys Res Commun 2008; 365: 1-7.

[5] Eggeling C, Ringemann C, Medda R, et al. Direct observation of the nanoscale dynamics of membrane lipids in a living cell. Nature 2009; 457: 1159-62.

[6] Pike LJ, Casey L. Cholesterol levels modulate EGF receptormediated signaling by altering receptor function and trafficking. Biochemistry 2002; 41: 10315-22.

[7] Jans R, Atanasova G, Jadot M, Poumay Y. Cholesterol depletion upregulates involucrin expression in epidermal keratinocytes through activation of p38. J Invest Dermatol 2004; 123: 564-73.

[8] Chen HW, Kandutsch AA, Heiniger HJ. The role of cholesterol in malignancy. Prog Exp Tumor Res 1978; 22: 275-316.

[9] Black HS, Herd JA, Goldberg LH, et al. Effect of a low-fat diet on the incidence of actinic keratosis. N Engl J Med 1994; 330: 1272-5.

[10] Vural P, Canbaz M, Sekçuki D, Murat A. Lipid profile in actinic keratosis and basal cell carcinoma. Int J Dermatol 1999; 38: 439-42

[11] Kornblau SM, Banker DE, Stirewalt D, et al. Blockade of adaptive defensive changes in cholesterol uptake and synthesis in AML by the addition of pravastatin to idarubicin + high-dose Ara-C: a phase 1 study. Blood 2007; 109: 2999-3006.

[12] Bang B, Gniadecki R, Gajkowska B. Disruption of lipid rafts causes apoptotic cell death in HaCaT keratinocytes. Exp Dermatol 2005; 14: 266-72.

[13] Gniadecki R. Depletion of membrane cholesterol causes ligandindependent activation of Fas and apoptosis. Biochem Biophys Res Commun 2004; 320: 165-9.
[14] Li YC, Park MJ, Ye SK, Kim CW, Kim YN. Elevated levels of cholesterol-rich lipid rafts in cancer cells are correlated with apoptosis sensitivity induced by cholesterol-depleting agents. Am J Pathol 2006; 168: 1107-18.

[15] Mollinedo F, Gajate C. Fas/CD95 death receptor and lipid rafts: new targets for apoptosis-directed cancer therapy. Drug Resist Update 2006; 9: 51-73.

[16] Legembre P, Daburon S, Moreau P, et al. Amplification of Fasmediated apoptosis in type II cells via microdomain recruitment. Mol Cell Biol 2005; 25: 6811-20.

[17] Gajate C, Gonzalez-Camacho F, Mollinedo F. Lipid raft connection between extrinsic and intrinsic apoptotic pathways. Biochem Biophys Res Commun 2009; 380: 780-4.

[18] Gajate C, Del Canto-Jañez E, Acuña AU, et al. Intracellular triggering of Fas aggregation and recruitment of apoptotic molecules into Fas-enriched rafts in selective tumor cell apoptosis. J Exp Med 2004; 200: 353-65.

[19] Bionda C, Athias A, Poncet D, et al. Differential regulation of cell death in head and neck cell carcinoma through alteration of cholesterol levels in lipid rafts microdomains. Biochem Pharmacol 2008; 75: 761-72.

[20] Datta SR, Brunet A, Greenberg ME. Cellular survival: a play in three Akts. Genes Dev 1999; 13: 2905-27.

[21] Shaw RJ, Cantley LC. Ras, PI(3)K and mTOR signalling controls tumour cell growth. Nature 2006; 441: 424-30.

[22] Rodriguez-Viciana $\mathrm{P}$, Warne $\mathrm{PH}$, Dhand $\mathrm{R}$, et al. Phosphatidylinositol-3-OH kinase as a direct target of Ras. Nature 1994; 370: 527-32.

[23] Burgering BM, Coffer PJ. Protein kinase B (c-Akt) in phosphatidylinositol-3-OH kinase signal transduction. Nature 1995; 376: 599-602.

[24] Harlan JE, Hajduk PJ, Yoon HS, Fesik SW. Pleckstrin homology domains bind to phosphatidylinositol-4,5-bisphosphate. Nature 1994; 371: 168-70.

[25] McManus EJ, Collins BJ, Ashby PR, et al. The in vivo role of PtdIns $(3,4,5) \mathrm{P} 3$ binding to PDK1 PH domain defined by knockin mutation. EMBO J 2004; 23: 2071-82.

[26] Alessi DR, James SR, Downes CP, et al. Characterization of a 3phosphoinositide-dependent protein kinase which phosphorylates and activates protein kinase B $\alpha$. Curr Biol 1997; 7: 261-9.

[27] Burnett PE, Barrow RK, Cohen NA, Snyder SH, Sabatini DM. RAFT1 phosphorylation of the translational regulators p70 S6 kinase and 4E-BP1. Proc Natl Acad Sci USA 1998; 95: 1432-7.

[28] Nave BT, Ouwens M, Withers DJ, Alessi DR, Shepherd PR. Mammalian target of rapamycin is a direct target for protein kinase B: identification of a convergence point for opposing effects of insulin and amino-acid deficiency on protein translation. Biochem J 1999; 344 Pt 2: 427-31.

[29] Bonni A, Brunet A, West AE, Datta SR, Takasu MA, Greenberg ME. Cell survival promoted by the Ras-MAPK signaling pathway by transcription-dependent and -independent mechanisms. Science 1999; 286: 1358-62.

[30] Greer EL, Brunet A. FOXO transcription factors at the interface between longevity and tumor suppression. Oncogene 2005; 24: 7410-25.

[31] Hope HR, Pike LJ. Phosphoinositides and phosphoinositideutilizing enzymes in detergent-insoluble lipid domains. Mol Biol Cell 1996; 7: 843-51.

[32] Lasserre R, Guo XJ, Conchonaud F, et al. Raft nanodomains contribute to Akt/PKB plasma membrane recruitment and activation. Nat Chem Biol 2008; 4: 538-47.

[33] Gao X, Zhang J. Spatiotemporal analysis of differential Akt regulation in plasma membrane microdomains. Mol Biol Cell 2008; 19: 4366-73.

[34] Altomare DA, Testa JR. Perturbations of the AKT signaling pathway in human cancer. Oncogene 2005; 24: 7455-64. 